\title{
The Cauchy Problem for First Order Partial Differential Equations
}

\section{AVNER FRIEDMAN}

\author{
Communicated by EBerhard HopF
}

1. Introduction. The Cauchy problem for quasi-linear equations

$$
u_{t}+\sum_{i=1}^{n} \frac{\partial}{\partial x_{i}} \phi_{i}(t, x, u)+\psi(t, x, u)=0 \quad(0<t \leqq T),
$$

with initial data on $t=0$, has been treated by many authors following the work of E. Hopf [9] who studied the special equation

$$
u_{t}+u u_{x}=0 \text {. }
$$

We refer, in particular, to a recent paper by Kruzkov [10] in which he established the existence of a bounded measurable weak solution and proved that it is uniquely determined by the "entropy condition." This condition was first discovered by Oleinik [12] for the case $n=1$. As noted in [12], already for the equation (1.2) the following is true: For any $\epsilon>0$ there are bounded smooth data with bounded derivatives for which no weak solution in the strip $0<t<\epsilon$ is continuous.

Kruzkov [10] assumed, for the proofs of existence and uniqueness, that

$$
-\chi_{u}(t, x, u) \leqq c_{0} \quad\left(c_{0} \text { constant }\right)
$$

where

$$
\chi=\sum_{i=1}^{n} \frac{\partial \phi_{i}}{\partial x_{i}}+\psi
$$

and, for any $R>0$, if $0 \leqq t \leqq T, x \varepsilon \mathrm{R}^{n}$ and $|u| \leqq R$, then

$$
|\chi(t, x, u)| \leqq \gamma_{R}, \quad\left|\frac{\partial \phi_{i}(t, x, u)}{\partial u}\right| \leqq \gamma_{R},
$$

where $\gamma_{R}$ is a constant.

Fleming [5] has considered the Cauchy problem for the nonlinear equation 


$$
u_{t}+F\left(t, x, u, u_{x}\right)=0 \quad(0 \leqq t<T)
$$

where $u_{x}=\left(\partial u / \partial x_{1}, \cdots, \partial u / \partial x_{n}\right)$; it is convenient here to impose the terminal condition (rather than an initial condition)

$$
u(T, x)=\phi(x) \quad\left(x \varepsilon \mathrm{R}^{n}\right) .
$$

He assumed that

$$
\begin{aligned}
& |F(t, x, 0,0)| \leqq C, \quad|F(t, x, u, p)-F(t, \bar{x}, u, p)| \leqq C|x-\bar{x}|, \\
& |F(t, x, u, p)-F(t, x, \bar{u}, p)| \leqq C|u-\bar{u}| \\
& |F(t, x, u, p)-F(t, x, u, \bar{p})| \leqq C|p-\bar{p}| \\
& |F(t, x, u, p)-F(\bar{t}, x, u, p)| \leqq C(1+|u|+|p|)|t-\bar{t}|^{\mu}
\end{aligned}
$$

for some positive constants $C, \mu$, and that $\phi$ is bounded and uniformly Lipschitz continuous. He then constructed a bounded and uniformly Lipschitz continuous generalized solution of (1.3), (1.4) by the method of "vanishing viscosity." (The same method is also used for the Cauchy problem of (1.1); see [10], [12].) Thus, he considered, for $\epsilon>0$, the parabolic Cauchy problem for

$$
\epsilon \triangle u+u_{t}+F\left(t, x, u, u_{x}\right)=0 \quad(0 \leqq t<T),
$$

under the terminal condition (1.4), and proved that the bounded solution $u^{\epsilon}$ converges to a generalized solution $u$ of (1.3), (1.4). This limit $u$ is the lower value of a certain differential game.

Notice that the equation (1.1) reduces to the equation of (1.3), with

$$
F(t, x, u, p)=\sum_{i=1}^{n} \frac{\partial \phi_{i}}{\partial x_{i}}+\psi+\sum_{i=1}^{n} \frac{\partial \phi_{i}}{\partial u} p_{i} .
$$

However, the restrictions in (1.5) are such that even the simple equation (1.2) is not included in Fleming's treatment.

Both Kruzkov [10] and Fleming [5] use the method of "vanishing viscosity" to construct their respective generalized solutions. In [10] the solution is generally discontinuous, whereas in [5] it is Lipschitz continuous. This phenomenon occurs even if the data are smooth [12]. It motivated us to find out what are the crucial restrictions on the $F$ in (1.3) which account for the Lipschitz continuity of the generalized solution obtained by the "vanishing viscosity" method.

Thus, our purpose in this paper is to generalize Fleming's results by relaxing the conditions in (1.5), but at the same time obtaining a Lipschitz continuous solution by the method of "vanishing viscosity." We shall, in fact, replace (1.5) by:

$$
\begin{aligned}
& F(t, x, 0,0) \leqq \gamma, \quad F(t, x, u, 0)-F(t, x, \bar{u}, 0) \leqq \gamma(u-\bar{u}) \text { if } u>\bar{u} \\
& F(t, x, u, p)-F(t, x, \bar{u}, p) \leqq \gamma_{R}(u-\bar{u}) \quad \text { if } \quad|u| \leqq R,|\bar{u}| \leqq R, u>\bar{u} \\
& |F(t, x, u, p)-F(t, \bar{x}, u, p)| \leqq \gamma_{R}(1+|p|)|x-\bar{x}| \text { if }|u| \leqq R
\end{aligned}
$$




$$
|F(t, x, u, p)-F(t, x, u, \bar{p})| \leqq \gamma_{R}(1+|x|)|p-\bar{p}| \text { if }|u| \leqq R,
$$

for any $R>0$ and $0 \leqq t \leqq T, x \varepsilon \mathbf{R}^{n}, \bar{x} \varepsilon \mathbf{R}^{n}, p \varepsilon \mathbf{R}^{n}, \bar{p} \varepsilon \mathbf{R}^{n}$, where $\gamma, \gamma_{R}$ are positive constants.

From (1.8)-(1.11) it follows that almost everywhere in $(t, x, u, p)$,

$$
\begin{gathered}
F(t, x, 0,0) \leqq \gamma, \quad F_{u}(t, x, u, 0) \leqq \gamma, \\
F_{u}(t, x, u, p) \leqq \gamma_{R} \quad \text { if }|u| \leqq R, \\
\left|F_{x}(t, x, u, p)\right| \leqq \gamma_{R}(1+|p|) \text { if }|u| \leqq R, \\
\left|F_{p}(t, x, u, p)\right| \leqq \gamma_{R}(1+|x|) \text { if }|u| \leqq R .
\end{gathered}
$$

If $F(t, x, u, p)$ is continuously differentiable with respect to $(x, u, p)$ then the sets of conditions (1.8)-(1.11) and $\left(1.8^{\prime}\right)-\left(1.11^{\prime}\right)$ are equivalent.

Comparing these conditions with those of Kruzkov (cited above), we see that the crucial restriction which we impose, and he does not, is (1.9). [When in (1.7) $F=F(u, p)$, this is the only restriction which we impose but Kruzkov does not require.]

Notice that the condition (1.9) for (1.1) implies that the $\partial \phi_{i} / \partial u$ are independent of $u$.

In Section 2 we establish the existence and uniqueness of a bounded solution $u^{\epsilon}$ of (1.6), (1.4), and derive an a priori bound on its first $x$-derivatives; this bound is independent of $\epsilon$.

In Section 3 we prove the existence of a "domain of dependence" for $\lim _{\epsilon \rightarrow 0} u^{\epsilon}$, provided the limit exists.

In Section 4 we consider a sequence of truncations $F^{m}$ of $F$. We apply Fleming's results to the Cauchy problem corresponding to $F^{m}$. Using the results of Sections 2, 3 we then establish the existence of a Lipschitz continuous generalized solution of (1.3), (1.4). Under the additional condition (2.15), the solution is obtained as $\lim u^{\epsilon}$.

Finally, in Section 5 we derive uniqueness theorems.

Added in proof. For the equation $n_{t}+f\left(\operatorname{grad}_{x} n\right)=0$, E. Hopf has constructed locally Lipschitz continuous solutions by fairly explicit formulas, in case either (i) $f$ is strictly convex in $\mathrm{R}^{n}$ and $f(n) /|n| \rightarrow \infty$ if $|n| \rightarrow \infty$, or (ii) $n(x, 0)$ is convex and $f(n)$ is continuous; see E. Hopf, Generalized solutions of nonlinear equations of first order, J. Math. Mech. 14 (1965), 951-974.

2. Existence and estimates for $u^{\epsilon}$. We shall need the following assumptions:

(A) $F(t, x, u, p)$ is uniformly Lipschitz continuous in $(x, u, p)$ and uniformly Hölder continuous in $t$ in compact subsets of $[0, T] \times \mathrm{R}^{n} \times \mathrm{R}^{1} \times \mathrm{R}^{n}$, and (1.8)-(1.11) hold for any $R>0$, where $\gamma, \gamma_{R}$ are positive constants.

(B) $\phi(x)$ is uniformly Lipschitz continuous, and

$$
|\phi(x)| \leqq \hat{\gamma}, \quad|\phi(x)-\phi(\bar{x})| \leqq \hat{\gamma}|x-\bar{x}| \quad(\hat{\gamma} \text { constant })
$$


for all $x \varepsilon \mathrm{R}^{n}, \bar{x} \varepsilon \mathbf{R}^{n}$.

Theorem 2.1. Let $(A),(B)$ hold. Then, for any $0<\epsilon<1$, there exists a unique bounded solution $u^{\epsilon}$ of (1.6), (1.4). Furthermore, there exist positive constants $K_{0}, K$, independent of $\epsilon$, such that

$$
\begin{aligned}
\left|u^{\epsilon}(t, x)\right| & \leqq K_{0}, \\
\left|\nabla_{x} u^{\epsilon}(t, x)\right| & \leqq K
\end{aligned}
$$

for all $0 \leqq t<T, x \varepsilon \mathrm{R}^{n}$.

Remark. From the proof of uniqueness given below it follows that the uniqueness holds in the larger class of functions $v(t, x)$ satisfying

$$
|v(t, x)| \leqq C e^{c|x|^{2}} \quad\left(0 \leqq t \leqq T, x \varepsilon \mathbf{R}^{n}\right)
$$

where $C, c$ are positive constants.

Proof. We first prove uniqueness. Suppose $u^{\epsilon}$ and $v^{\epsilon}$ are both bounded solutions of (1.6), (1.4) and set $z=u^{\epsilon}-v^{\epsilon}$. Then

$$
\epsilon \Delta z+z_{t}+\widetilde{F}_{u} z+\sum_{i=1}^{n} \widetilde{F}_{p_{i}} z_{x_{i}}=0 \text { if } \quad 0<t<T,
$$

$z=0$ on $t=T$, and $z(t, x)$ is a bounded function. The coefficients $\widetilde{F}_{u}, \widetilde{F}_{p_{i}}$ are functions of $(t, x)$, and $\widetilde{F}_{u} \leqq \gamma$. Further, since $u^{\epsilon}$ and $v^{\epsilon}$ are bounded, the condition (1.11) implies that

$$
\left|\widetilde{F}_{p_{i}}\right| \leqq \text { const. }(1+|x|) .
$$

From [6; p. 43, Theorem 9] it then follows that $z \equiv 0$.

In order to prove the existence of a bounded solution $u^{\epsilon}$, we first assume:

$$
|F(t, x, u, p)| \leqq N \quad(N \text { constant }),
$$

(2.5) $\quad F$ and $\phi$ have first continuous derivatives in all their arguments.

For any positive integer $m$, let $\psi_{m}(t, x)$ be a continuous extension of $\phi(x)$ into the set $0 \leqq t \leqq T,|x|=m$, such that $\left|\psi_{m}\right| \leqq \hat{\gamma}$. Denote by $u_{m}^{\epsilon}$ the solution of (1.6) in the region $0 \leqq t<T,|x|<m$, satisfying the terminal-boundary conditions:

$$
\begin{gathered}
u_{m}^{\epsilon}(T, x)=\phi(x) \text { if }|x| \leqq m, \\
u_{m}^{e}=\psi_{m} \text { if } 0 \leqq t \leqq T, \quad|x|=m .
\end{gathered}
$$

The existence of $u_{m}^{\epsilon}$ follows by standard theorems (see, for instance, [6] or [11]). We shall prove that

$$
\left|u_{m}^{\epsilon}(t, x)\right| \leqq(\hat{\gamma}+1)^{\delta(T-t)}
$$

where $\delta$ is a positive constant independent of $\epsilon, m, N$.

Let $w=(\hat{\gamma}+1) e^{\delta(T-t)}$. Then 


$$
\begin{aligned}
\epsilon \Delta w+w_{\imath}+F\left(t, x, w, w_{x}\right) & =-\delta w+F(t, x, w, 0) \\
& =-\delta w+F(t, x, 0,0)+w F_{u}(t, x, w, 0)
\end{aligned}
$$

for some $w_{\varepsilon}(0, w)$. By $\left(1.8^{\prime}\right)$, the right hand side is negative if

$$
\delta>\gamma+\gamma /(\hat{\gamma}+1) .
$$

Since also $w>u_{m}^{e}$ on $t=T$ and on $|x|=m$, we can employ a standard comparison theorem [6; p. 52] and conclude that $w>u_{m}^{e}$ if $0 \leqq t \leqq T,|x| \leqq m$. Similarly one shows that $w>-u_{m}^{\epsilon}$; hence the assertion (2.6).

Since $(2.4)$ holds, we can apply the a priori $(1+\delta)$-estimates of [6] in order to establish a uniform Hölder condition on $u_{m}^{\epsilon}$ in compact subsets of $0 \leqq t \leqq T$, $x \varepsilon \mathrm{R}^{n}$; a uniform Hölder condition on $\nabla_{x} u_{m}^{\epsilon}$ in compact subsets of $0 \leqq t<T$, $x \in \mathrm{R}^{n}$, and the bound

$$
\left|\nabla_{x} u_{m}^{e}\right| \leqq C_{\epsilon, N} \quad(0 \leqq t \leqq T,|x| \leqq m-1)
$$

where $C_{\epsilon, N}$ is a constant depending on $\epsilon, N$, but independent of $m$.

Using the Hölder continuity of $u_{m}^{\epsilon}, \nabla_{x} u_{m}^{\epsilon}$, we can then employ the interior Schauder estimates [6] in order to obtain a uniform Hölder condition in compact subsets of the strip $0 \leqq t<T$ for $D_{x}^{2} u_{m}^{\epsilon}, D_{t} u_{m}^{\epsilon}$. But then, by compactness, there is a subsequence of $m$ 's for which $u_{m}^{\epsilon}$ is convergent to a solution $u^{\epsilon}$ of (1.6), (1.4), uniformly on compact subsets. From (2.6), (2.7) we further obtain

$$
\begin{gathered}
\left|u^{\epsilon}(t, x)\right| \leqq(\hat{\gamma}+1) e^{\delta T}, \\
\left|\nabla_{x} u^{\epsilon}(t, x)\right| \leqq C_{\epsilon, N} .
\end{gathered}
$$

We next improve the estimate (2.9). Let

$$
v^{\lambda}(t, x)=\frac{\partial u^{\epsilon}(t, x)}{\partial x_{\lambda}} \quad(\lambda=1, \cdots, n) .
$$

Since $F$ is in $C^{1}$, we may differentiate (1.6) with respect to $x_{\lambda}$ and obtain

$$
\epsilon \Delta v^{\lambda}+v_{t}^{\lambda}+F_{x_{\lambda}}+F_{u} v^{\lambda}+\sum_{\mu=1}^{n} F_{p_{\mu}} \frac{\partial v^{\lambda}}{\partial x_{\mu}}=0
$$

the argument in $F_{x_{\lambda}}, F_{u}, F_{p_{\mu}}$ is $(t, x, u, v)$, where $v=\left(v^{1}, \cdots, v^{n}\right)$. Here we have used the fact that

$$
\frac{\partial v^{\mu}}{\partial x_{\lambda}}=\frac{\partial^{2} u^{\epsilon}}{\partial x_{\lambda} \partial x_{\mu}}=\frac{\partial v^{\lambda}}{\partial x_{\mu}}
$$

On $t=T, v^{\lambda}=\partial \phi / \partial x_{\lambda}$.

Multiplying both sides of (2.10) by $2 v^{\lambda}$, summing over $\lambda$, and using the inequality

$$
2 v^{\lambda} \Delta v^{\lambda}=\Delta\left(v^{\lambda}\right)^{2}-2 \nabla_{x} v^{\lambda} \cdot \nabla_{x} v^{\lambda} \leqq \triangle\left(v^{\lambda}\right)^{2},
$$

we deduce, for $w=\sum_{\lambda=1}^{n}\left(v^{\lambda}\right)^{2}$, the inequality 


$$
\epsilon \triangle w+w_{t}+2 F_{x} \cdot v+2 F_{u} w+\sum_{\mu=1}^{n} F_{p_{\mu}} \frac{\partial w}{\partial x_{\mu}} \geqq 0 .
$$

From (2.8), (1.10') it follows that

$$
2\left|F_{x} \cdot v\right| \leqq \gamma^{\prime}(1+w)
$$

where $\gamma^{\prime}$ is a constant depending only on $\gamma_{R}$ for $R=(\hat{\gamma}+1) e^{\delta T}$. Using also $\left(1.9^{\prime}\right)$, we deduce from $(2.11)$ :

$$
\epsilon \Delta w+w_{t}+\gamma^{\prime}+3 \gamma^{\prime} w+\sum_{\mu=1}^{n} F_{p_{\mu}} \frac{\partial w}{\partial x_{\mu}} \geqq 0 .
$$

We shall compare $w$ with $z=n \hat{\gamma}^{2} e^{\eta(T-t)}$ for some $\eta>0$. Clearly,

$$
\epsilon \triangle z+z_{t}+\gamma^{\prime}+3 \gamma^{\prime} z+\sum_{\mu=1}^{n} F_{p_{\mu}}\left(t, x, u, u_{x}\right) \frac{\partial z}{\partial x_{\mu}}=z\left(-\eta+3 \gamma^{\prime}\right)+\gamma^{\prime}<0
$$

if

$$
\eta>3 \gamma^{\prime}+\gamma^{\prime} / n \hat{\gamma}^{2}
$$

Since $z(T)=n \hat{\gamma}^{2} \geqq w(T, x)$ and since by $(2.9),\left(1.11^{\prime}\right)$,

$$
\left|F_{p_{\mu}}\left(t, x, u, u_{x}\right)\right| \leqq \text { const. }(1+|x|),
$$

we conclude by $[6 ;$ p. 43 , Theorem 9$]$ that

$$
w(t, x) \leqq n \hat{\gamma}^{2} e^{\eta(T-t)} .
$$

So far we have proved the existence of $u^{\epsilon}$ and the inequalities (2.2), (2.3) under the additional assumptions (2.4), (2.5). The constants $K_{0}, K$, however, do not depend on $N$ (they depend on $\gamma, \hat{\gamma}$ and $\gamma_{R}$ for some $R$ depending only on $\gamma, \hat{\gamma})$.

In order to construct $u^{\epsilon}$ in general, consider a sequence of functions $F^{i}(t, x, u, p)$ satisfying $(A)$ and (2.4), (2.5) with $F=F^{i}, N=N_{i}$ such that the constants $\gamma$, $\gamma_{R}$ in (1.8)-(1.11) for $F=F^{i}$ are independent of $j$, and such that

$$
F^{i}(t, x, u, p) \rightarrow F(t, x, u, p) \text { as } j \rightarrow \infty,
$$

uniformly in compact subsets. We also introduce continuously differentiable functions $\phi^{i}(x)$ satisfying $(B)$ with a constant $\hat{\gamma}$ independent of $j$, such that

$$
\phi^{i}(x) \rightarrow \phi(x) \text { as } j \rightarrow \infty,
$$

uniformly in compact subsets. Denote by $u^{\epsilon, i}$ the bounded solution $u^{\epsilon}$ corresponding to $F^{i}, \phi^{i}$. By what we have proved above,

$$
\begin{aligned}
\left|u^{\epsilon, j}(t, x)\right| & \leqq K_{0}, \\
\left|\nabla_{x} u^{\epsilon, i}(t, x)\right| & \leqq K
\end{aligned}
$$

for all $j$ and for all $0 \leqq t<T, x \varepsilon \mathrm{R}^{n}$.

From $\left(1.10^{\prime}\right)$ for $F^{i}$ we deduce 


$$
\left|F^{i}(t, x, u, p)\right| \leqq \gamma_{R}(1+|p|)(1+|x|) \quad \text { if } \quad|u| \leqq R .
$$

Hence, by (2.13), (2.14),

$$
\left|F^{i}\left(t, x, u^{\epsilon, i}(t, x), \nabla_{x} u^{\epsilon, i}(t, x)\right)\right| \leqq C(1+|x|)
$$

where $C$ is a constant independent of $j$. This inequality enables us to use the $(1+\delta)$-estimates of [6] in order to deduce (i) a uniform Hölder condition for $u^{\epsilon, i}$ in compact subsets of $0 \leqq t \leqq T, x \varepsilon \mathrm{R}^{n}$, and (ii) a uniform Hölder condition for $\nabla_{x} u^{\epsilon, i}$ in compact subsets of $0 \leqq t<T, x \varepsilon \mathrm{R}^{n}$; the Hölder coefficients are independent of $j$. We can then employ the interior Schauder estimates [6] in order to derive a uniform Hölder condition in compact subsets of $0 \leqq t<T$, $x \varepsilon \mathrm{R}^{n}$ for $D_{x}^{2} u^{\epsilon, j}, D_{t} u^{\epsilon, j}$. The Hölder coefficients can be made to be independent of $j$ if we choose the $F^{i}(t, x, u, p)$ such that they are uniformly Hölder continuous in $(t, x, u, p)$ in compact subsets, uniformly with respect to $j$. (This can always be done.)

We can now employ a compactness argument to deduce that, for a subsequence of $j^{\prime} \mathrm{s}, u^{\epsilon, i}$ converges to a solution $u^{\epsilon}$ of (1.6), (1.4), uniformly on compact subsets of $0 \leqq t \leqq T, x \varepsilon \mathrm{R}^{n}$. The assertions (2.2), (2.3) follow from (2.13), (2.14).

Suppose now that

$$
|F(t, x, u, p)-F(\bar{t}, x, u, p)| \leqq \gamma_{R}(1+|p|)|t-\bar{t}| \quad \text { if } \quad|u| \leqq R,
$$

for any $R>0$ and for all $0 \leqq t \leqq T, 0 \leqq \bar{t} \leqq T, x \varepsilon \mathbf{R}^{n}, p \varepsilon \mathbf{R}^{n}$.

Suppose also that

$$
\begin{gathered}
\phi(x) \text { is in } C^{2} \text { and }\left|D_{x}^{2} \phi\right| \leqq \hat{\gamma}, \\
\left\{\begin{array}{l}
F(t, x, u, p) \text { is continuously differentiable, and } \\
|F|+\left|F_{t}\right|+\left|F_{x}\right|+\left|F_{u}\right|+\left|F_{p}\right| \leqq N .
\end{array}\right.
\end{gathered}
$$

Then we can differentiate (1.6) with respect to $t$ and derive, by a comparison argument, the inequality

$$
\left|D_{t} u^{\epsilon}(t, x)\right| \leqq K \quad\left(0 \leqq t \leqq T, x \varepsilon \mathrm{R}^{n}\right)
$$

where $K$ is a constant independent of $\epsilon, N$. By approximating a general $F$ (satisfying $(A),(2.15)$ ) by a sequence of $F^{i}$ satisfying also (2.17), we conclude:

Theorem 2.2. Let the conditions $(A),(B)$ and (2.15), (2.16) hold. Then the bounded solution $u^{\epsilon}$ of (1.6), (1.4) satisfies (2.2), (2.3) and (2.18).

\section{Domain of dependence.}

Lemma 3.1. Let the conditions $(A),(B)$ hold for a pair $F, \phi$ and for a pair $\tilde{F}, \boldsymbol{\Phi}$. Denote by $u^{\epsilon}$ and $\tilde{u}^{\epsilon}$ the corresponding solutions of (1.6), (1.4). Suppose that

$$
u(t, x)=\lim _{e \rightarrow 0} u^{e}(t, x), \quad \tilde{u}(t, x)=\lim _{\epsilon \rightarrow 0} \tilde{u}^{e}(t, x)
$$

exist for each $(t, x) \varepsilon[0, T] \times \mathrm{R}^{n}$. Suppose finally that, for some $\rho>0$, 


$$
\begin{aligned}
F(t, x, u, p) & =\widetilde{F}(t, x, u, p) \quad \text { if } \quad 0 \leqq t \leqq T,|x| \leqq \rho,|u| \leqq K_{0},|p| \leqq K, \\
\phi(x) & =\Phi(x) \text { if }|x| \leqq \rho,
\end{aligned}
$$

where $K_{0}, K$ are constants such that (by Theorem 2.1) (2.2) and (2.3) hold for both $u^{\epsilon}$ and $\tilde{u}^{\epsilon}$. Then

$$
u(t, x)=\tilde{u}(t, x) \text { if } 0 \leqq t \leqq T, \quad|x| \leqq c \rho
$$

where $c$ is a positive constant depending only on $T$.

Proof. If $0 \leqq t \leqq T,|x| \leqq \rho$, then

$$
\begin{aligned}
F\left(t, x, u^{\epsilon}(t, x), u_{x}^{\epsilon}(t, x)\right) & =\widetilde{F}\left(t, x, \tilde{u}^{\epsilon}(t, x), \tilde{u}_{x}^{\epsilon}(t, x)\right), \\
\phi(x) & =\Phi(x) .
\end{aligned}
$$

Hence, the function $v^{\epsilon}=u^{\epsilon}-\tilde{u}^{\epsilon}$ satisfies:

$$
\begin{aligned}
& \epsilon \Delta v^{\bullet}+v_{t}=0 \quad \text { if } \quad 0 \leqq t<T, \quad|x| \leqq \rho, \\
& v^{\epsilon}(T, x)=0 \quad \text { if } \quad|x| \leqq \rho, \\
& \left|v^{\epsilon}(t, x)\right| \leqq 2 K_{0} \quad \text { if } \quad 0 \leqq t \leqq T, \quad|x|=\rho .
\end{aligned}
$$

Consider, for $\lambda>0$, the function

$$
z(t, x)=2 K_{0} e^{\left[\lambda(T-t)+|x|^{2}-\rho^{2}\right] / \epsilon} .
$$

It satisfies:

$$
\begin{aligned}
& z(T, x)>0=v^{\epsilon}(T, x) \quad \text { if }|x| \leqq \rho, \\
& z(t, x)=2 K_{0} e^{\lambda(T-t) / \epsilon} \geqq 2 K_{0} \geqq v^{\epsilon}(t, x) \quad \text { if } \quad 0 \leqq t \leqq T,|x|_{1}=\rho .
\end{aligned}
$$

Further, if $0<t<T$, $|x|<\rho$,

$$
\epsilon \Delta z+z_{t}=\left[\epsilon\left(\frac{4|x|^{2}}{\epsilon^{2}}+\frac{2 n}{\epsilon}\right)-\frac{\lambda}{\epsilon}\right] z<0
$$

if $\lambda=5 \rho^{2}$ and $\epsilon$ is sufficiently small. Hence, by the maximum principle, $v^{*} \leqq z$ if $0 \leqq t \leqq T,|x| \leqq \rho$.

Similarly, $-v^{\epsilon} \leqq z$. Thus,

$$
\left|u^{\epsilon}(t, x)-\tilde{u}^{\epsilon}(t, x)\right| \leqq 2 K_{0} e^{\left[5 \rho^{2}(T-t)+|x|^{2}-\rho^{2}\right] / \epsilon}
$$

if $0 \leqq t \leqq T,|x| \leqq \rho$. Taking $\epsilon \rightarrow 0$ we conclude that

$$
u(t, x)=\tilde{u}(t, x) \text { if } \quad 0 \leqq T-t \leqq \frac{1}{10},|x|^{2} \leqq \frac{\rho^{2}}{2}
$$

Proceeding step by step, we deduce that (3.2) holds if

$$
0 \leqq T-t \leqq \mu,|x|^{2} \leqq \frac{\rho^{2}}{2^{i}} \quad(j=1,2, \cdots)
$$

for any $\mu \leqq j / 10, \mu \leqq T$. Taking $j$ such that $j / 10 \geqq T$, the assertion (3.1) follows. 


\section{Existence theorems.}

Definition. A function $u(t, x)$ which is uniformly Lipschitz continuous in $(t, x)$ in compact subsets of $0 \leqq t \leqq T, x \varepsilon \mathrm{R}^{n}$ is called a generalized solution of (1.3), (1.4) if it satisfies (1.3) almost everywhere and (1.4) everywhere.

We shall now construct a generalized solution of (1.3), (1.4) assuming that $(A),(B)$ hold.

For any positive integer $m$, truncate $F$ into $F^{m}$ so that

$$
\begin{aligned}
& \left|F^{m}(t, x, 0,0)\right| \leqq C_{m}, \quad\left|F^{m}(t, x, u, p)-F^{m}(t, \bar{x}, u, p)\right| \leqq C_{m}|x-\bar{x}|, \\
& \left|F^{m}(t, x, u, p)-F^{m}(\bar{t}, x, u, p)\right| \leqq C_{m}|t-\bar{t}| \\
& \left|F^{m}(t, x, u, p)-F^{m}(t, x, \bar{u}, p)\right| \leqq C_{m}|u-\bar{u}| \\
& \left|F^{m}(t, x, u, p)-F^{m}(t, x, u, \bar{p})\right| \leqq C_{m}|p-\bar{p}|,
\end{aligned}
$$

where $C_{m}$ is a constant depending on $m$, and

$$
F^{m}(t, x, u, p)=F(t, x, u, p) \text { if }|x|+|u|+|p|<m .
$$

We can accomplish such a truncation that the conditions $(A),(B)$ will hold for $F=F^{m}$ with constants $\gamma, \gamma_{R}$ independent of $m$.

Following Fleming [5], write $F^{m}$ as the lower Hamiltonian function $H^{m}$ of a differential game $G_{m}$ with dynamics

$$
\frac{d x}{d t}=f(t, x, y, z), \quad x\left(t_{0}\right)=x_{0},
$$

and payoff

$$
P(y, z)=\int_{t_{0}}^{T} h(t, x, y, z) d t+\left\{\exp \left[\int_{t_{0}}^{T} g(t, x, y, z) d t\right]\right\} \phi(x(T))
$$

Thus

$$
H^{m}(t, x, u, p)=\max _{y \geq Y} \min _{z \varepsilon Z}\{f(t, x, y, z) \cdot p+g(t, x, y, z) u+h(t, x, y, z)\}
$$

The control sets are

$$
\begin{aligned}
& Y=\left\{y=\left(y_{0}, y^{\prime}\right) ;\left|y_{0}\right| \leqq M_{0},\left|y^{\prime}\right| \leqq M^{\prime}\right\} \\
& Z=\left\{z=\left(z_{0}, z^{\prime}\right) ;\left|z_{0}\right| \leqq N_{0},\left|z^{\prime}\right| \leqq N^{\prime}\right\}
\end{aligned}
$$

where $y^{\prime}=\left(y_{1}, \cdots, y_{n}\right), z^{\prime}=\left(z_{1}, \cdots, z_{n}\right)$, and

$$
\begin{aligned}
& f(t, x, y, z)=\frac{F\left(t, x, y_{0}, y^{\prime}\right)}{1+\left|y^{\prime}\right|^{2}} y^{\prime}+z^{\prime} \\
& h(t, x, y, z)=\frac{F\left(t, x, y_{0}, y^{\prime}\right)}{1+\left|y^{\prime}\right|^{2}}-y_{0} z_{0}-y^{\prime} \cdot z^{\prime} \\
& g(t, x, y, z)=z_{0} .
\end{aligned}
$$


We take $M_{0}=K_{0}, M^{\prime}=K$ where $K_{0}, K$ are constants independent of $m$ such that (by Theorem 2.1) the bounded solution $u^{\epsilon, m}$ of (1.6), (1.4) with $F=F^{m}$ satisfies:

$$
\left|u^{\epsilon, m}\right| \leqq K_{0}, \quad\left|\nabla_{x} u^{\epsilon, m}\right| \leqq K .
$$

We further take $N_{0}=C_{m}$ and $N^{\prime}$ such that (cf. Lemma 2 of [5]) whenever $F(p)$ satisfies a Lipschitz condition with coefficient $C_{m}$ and $|F(0)| \leqq C_{m}\left(1+K_{0}\right)$, and

$$
F_{0}(p)=\frac{F(p)}{1+|p|^{2}}, \quad F_{i}(p)=\frac{F(p) p_{i}}{1+|p|^{2}} \quad(i=1, \cdots, n)
$$

then the inequality

$$
\left|F_{0}(q)-F_{0}(p)+\sum_{i=1}^{n}\left[F_{i}(q)-F_{i}(p)\right] p_{i}\right| \leqq N^{\prime}|q-p|
$$

holds. Thus, $N^{\prime}=N^{\prime}(m)$.

As shown in [5], for all $0 \leqq t \leqq T, x \varepsilon \mathrm{R}^{n}$,

$$
H^{m}(t, x, u, p)=F^{m}(t, x, u, p) \quad \text { if } \quad|u| \leqq K_{0}, \quad|p| \leqq K .
$$

By introducing "white noise" $2 \epsilon^{1 / 2} \nu(t)$ (where $\nu(t)$ has the first two moments of $d w / d t ; w(t)$ Brownian motion) one obtains (see [5]) a stochastic game whose lower value $W^{\epsilon, m}$ is a bounded solution of (1.6), (1.4) with $F=H^{m}$, i.e.,

$$
\left\{\begin{array}{c}
\epsilon W^{\epsilon, m}+\frac{\partial}{\partial t} W^{\epsilon, m}+H^{m}\left(t, x, W^{\epsilon, m}, \nabla_{x} W^{\epsilon, m}\right)=0 \quad(0 \leqq t<T), \\
W^{\epsilon, m}(T, x)=\phi(x) .
\end{array}\right.
$$

Finally (by [4], [5]), the lower value $V^{m}(t, x)$ of the game $G_{m}$ (with initial point $(t, x))$ is the uniform limit, in compact sets, of $W^{\epsilon, m}(t, x)$ as $\epsilon \rightarrow 0$.

Since $F=H^{m}$ satisfies the conditions in $(A), W^{\epsilon, m}$ is the unique bounded solution of (4.8). Since, further, the unique bounded solution $u^{\epsilon, m}$ of (1.6), (1.4) with $F=F^{m}$ satisfies (4.6), and since (4.7) holds, $u^{\epsilon, m}$ is also a bounded solution of (4.8). Hence

$$
u^{\epsilon, m}=W^{\epsilon, m}
$$

It follows that

$$
\lim _{\epsilon \rightarrow 0} u^{\epsilon, m}(t, x)=V^{m}(t, x) .
$$

Similarly, for any other positive integer $m^{\prime}$,

$$
\lim _{\epsilon \rightarrow 0} u^{e, m^{\prime}}(t, x)=V^{m^{\prime}}(t, x)
$$

where $V^{m^{\prime}}$ is the value of $G_{m}$.

Using Lemma 3.1 we deduce that if $m^{\prime}>m$, then 


$$
V^{m^{\prime}}(t, x)=V^{m}(t, x) \quad \text { if } \quad 0 \leqq t \leqq T, \quad|x| \leqq c m
$$

where $c$ is a positive constant independent of $m$.

Consider the function

$$
u(t, x)=\lim _{m \rightarrow \infty} V^{m}(t, x) .
$$

For each $R>0, u(t, x)$ is the lower value of the game $G_{m}$ initiating at $(t, x)$, where $0 \leqq t \leqq T,|x| \leqq R$, provided $m$ is sufficiently large.

As shown by Fleming [ 5 ; p. 106], $V^{m}(t, x)$ is uniformly Lipschitz continuous (the Lipschitz continuity with respect to $x$ follows also from (4.6)), and it satisfies the equation

$$
\frac{\partial V^{m}}{\partial t}+H^{m}\left(t, x, V^{m}, \nabla_{x} V^{m}\right)=0
$$

almost everywhere. It follows that $u(t, x)$ is uniformly Lipschitz continuous, and it satisfies

$$
\frac{\partial u}{\partial t}+H^{m}\left(t, x, u, \nabla_{x} u\right)=0
$$

almost everywhere when $0 \leqq t \leqq T,|x| \leqq R$, provided $m$ is sufficiently large. From (4.12), (4.10), (4.6) we deduce that $|u| \leqq K_{0}$ and $\left|u_{x}\right| \leqq K$ almost everywhere. Recalling (4.7), (4.2), we see that $u$ satisfies (1.3) almost everywhere. We have thus proved:

Theorem 4.1. Let $(A),(B)$ hold. Then there exists a bounded and uniformly Lipschitz continuous function $u(t, x)$ which is a generalized solution of (1.3), (1.4). For any $R>0$, there is an $m_{0}=m_{0}(R)>0$ such that $u(t, x)$ coincides with the lower value of the game $G_{m}$ initiating at $(t, x)$, where $0 \leqq t \leqq T,|x| \leqq R$, provided $m \geqq m_{0}(R)$.

Assume now, in addition to $(A),(B)$ that (2.15), (2.16) hold. Then, by Theorem 2.2,

$$
\left|D_{t} u^{\epsilon}(t, x)\right| \leqq K^{\prime} \quad\left(0 \leqq t<T, x \varepsilon \mathrm{R}^{n}\right)
$$

where $K^{\prime}$ is a constant independent of $\epsilon$.

From (4.13), and (2.2), (2.3) we conclude that, for any compact subset $S$ of $\{0 \leqq t \leqq T\} \times \mathrm{R}^{n}$, the family $\left\{u^{\epsilon} ; 0<\epsilon<1\right\}$ is uniformly bounded and equicontinuous on $S$. If for a sequence of $\left\{\epsilon_{i}\right\}$ converging to zero, $\left\{u^{\epsilon^{i}}\right\}$ is uniformly convergent on $S$, then (by Lemma 3.1 with $\epsilon$ replaced by $\epsilon_{i}$ ) the limit must coincide, on $S$, with $V^{m}$ provided $m$ is sufficiently large. Consequently, $\lim u^{\epsilon_{i}}$ is independent of the particular sequence $\epsilon_{j}$. This implies that

$$
u(t, x)=\lim _{\epsilon \rightarrow 0} u^{\epsilon}(t, x)
$$

exists, uniformly on each set $0 \leqq t \leqq T,|x| \leqq R$, and $u(t, x)$ coincides with $V^{m}(t, x)$ if $m$ is sufficiently large, depending on $R$; say if $m \geqq m^{*}(R)$. 
We shall now prove:

Theorem 4.2. Let $(A),(B)$ and (2.15) hold and denote by $u^{\epsilon}(t, x)$ the bounded solution of (1.6), (1.4). Then the limit in (4.14) exists uniformly on compact subsets, and $u(t, x)$ is the generalized solution of (1.3), (1.4) constructed in Theorem 4.1 .

Proof. We have already proved the theorem in case (2.16) is also satisfied. Now let $\phi_{i}$ be functions satisfying (2.16) with $\hat{\gamma}=\hat{\gamma}_{i}$, for which (2.1) holds with $\hat{\gamma}$ independent of $j$, and such that

$$
\sup _{\mathrm{R}^{n}}\left|\phi_{j}(x)-\phi(x)\right| \leqq \delta_{i} \rightarrow 0 .
$$

Denote by $u_{i}^{e}$ the bounded solution $u^{e}$ of (1.6), (1.4) corresponding to $\phi_{i}$, and denote by $u_{i}$ the generalized solution of (1.3), (1.4) constructed above for $\phi=\phi_{j}$. Thus,

$$
\lim _{\epsilon \rightarrow 0}\left|u_{j}^{\epsilon}(t, x)-u_{j}(t, x)\right|=0
$$

uniformly on compact subsets. Comparing $u_{j}^{e}-u_{i}$ with $c \delta_{j} e^{\mu(T-t)}$ (where $c, \mu$ are positive constants) by means of Theorem 9 of [6; p. 43], we get

$$
\left|u_{\boldsymbol{i}}^{\bullet}(t, x)-u^{\epsilon}(t, x)\right| \leqq C \delta_{i}
$$

where $C$ is a constant independent of $\epsilon, j$.

For fixed $R>0$, if $0 \leqq t \leqq T,|x| \leqq R$, then $u(t, x)$ and $u_{j}(t, x)$ coincide with the lower values of the games $G_{m}\left(m \geqq m^{*}(R)\right)$, initiating at $(t, x)$, corresponding to $\phi$ and $\phi_{i}$ respectively. Since (4.15) holds, one easily deduces that

$$
\lim _{j \rightarrow \infty}\left|u(t, x)-u_{j}(t, x)\right|=0
$$

uniformly for $0 \leqq t \leqq T,|x| \leqq R$.

Now, for any $j$,

$$
\begin{aligned}
\varlimsup_{\epsilon \rightarrow 0}\left|u^{e}-u\right| & \leqq \varlimsup_{\epsilon \rightarrow 0}\left|u^{e}-u_{i}^{e}\right|+\varlimsup_{\epsilon \rightarrow 0}\left|u_{i}^{e}-u_{i}\right|+\left|u_{i}-u\right| \\
& \leqq 0+C \delta_{i}+\left|u_{i}-u\right|
\end{aligned}
$$

by (4.16), (4.17). Taking $j \rightarrow \infty$ and using (4.18), we get the assertion (4.14), uniformly for $0 \leqq t \leqq T,|x| \leqq R$. This completes the proof of the theorem.

\section{Uniqueness.}

Theorem 5.1. Let the conditions $(A),(B)$ hold. If $u$ is a bounded $C^{1}$ solution of (1.3), (1.4), and if $\nabla_{x} u$ is bounded, then $u$ is uniquely determined.

Proof. Consider the truncation $F^{m}$ employed in the proof of Theorem 4.1. Then $u$ is a $C^{1}$ solution of the equation 


$$
\frac{\partial u}{\partial t}+H^{m}\left(t, x, u, u_{x}\right)=0
$$

for $0 \leqq t \leqq T,|x| \leqq R$, provided $m$ is sufficiently large, say $m \geqq m^{*}(R)$. But then, by a result regarding comparison theorems for differential games ([7], [3], [8]) $u(t, x)$ must coincide with the lower value $V^{m}(t, x)$ if $0 \leqq t \leqq T,|x| \leqq R^{\prime}$, where $R^{\prime}(<R)$ is related to $R$, and $R^{\prime}$ can be made arbitrarily large if $R$ is sufficiently large. It follows that $u$ is uniquely determined.

We mention (see, for instance, [1]) that if $F \varepsilon C^{2}, \phi \varepsilon C^{2}$, then any $C^{2}$ solution of (1.3), (1.4) is uniquely determined by the method of characteristics. For $n=1$, uniqueness theorems under weaker differentiability assumptions were proved by Haar [1; pp. 145-147] and A. Douglis [2].

Definition. We say that $u(t, x)$ satisfies the "differential game condition" if for any compact set $0 \leqq t \leqq T,|x| \leqq R, u(t, x)$ coincides with the lower value $V^{m}(t, x)$ of the game $G_{m}$ initiating at $(t, x)$, for all $m$ sufficiently large (depending on $R$ ).

This concept (like the "entropy condition") can actually be expressed in terms of test functions. However the test functions are controls $y(t), z(t) \mathrm{em}-$ ployed by the two players. We also mention that, in view of Theorem 5.1, every bounded function $u$ with bounded continuous derivatives, which satisfies (1.3), (1.4), must satisfy the "differential game condition." Finally, we can trivially state:

Theorem 5.2. Let $(A),(B)$ hold. Then there exists a unique generalized solution of (1.3), (1.4) satisfying the "differential game condition."

\section{REFERENCES}

1. R. Courant \& D. Hilbert, Methods of mathematical physics, vol. II., Partial differential equations, Interscience Publishers, John Wiley and Sons, New York, 1962.

2. A. DouguIs, The continuous dependence of generalized solutions on nonlinear partial differential equations upon initial data, Comm. Pure Appl. Math. 14 (1961), 267-284.

3. R. J. Euliott \& N. J. Kalton, Cauchy problems for certain Isaacs-Bellman equations and games of survival, (to appear).

4. W. H. Fleming, The convergence problem for differential games II, Advances in game theory, Annals of Math. Studies, No. 52, (1964), 195-210.

5. W. H. Fleming, The Cauchy problem for degenerate parabolic equations, J. Math. Mech. 13 (1964), 987-1008.

6. A. Friedman, Partial differential equations of parabolic type, Prentice-Hall, Englewood Cliffs, N. J., 1964.

7. A. Friedman, Comparison theorems for differential games, I, J. Diff. Eqs., 12 (1972), 162-172.

8. A. Friedman, Remarks on differential games of survival, J. Diff. Eqs. (to appear).

9. E. Hopf, The partial differential equation $u_{t}+u u_{x}=\mu u_{x x}$, Comm. Pure Appl. Math. 3 (1950), 201-230.

10. S. N. KRUzKov, First order quasilinear equations in several independent variables, Math. USSR-Sb. 10 (1970), 217-243 [Math. Sbornik 81 (123)(1970), no. 2]. 
11. O. A. Ladyzenskaja, V. A. Solonnikov \& N. N. Ural'ceva, Linear and quasilinear equations of parabolic type, Amer. Math. Soc., Providence, R. I., 1968.

12. O. A. Oleinik, Discontinuous solutions on nonlinear differential equations, Usp. Math. Nauk 12 (1957), no. 3 (75), 3-73; Amer. Math. Soc. Transl. (2) 26 (1963), 95-172.

This work was partially supported by National Science Foundation Grant GP-35347X.

Northwestern University

Date communicated: OCTOBER 30, 1972 